OBSERVATIONAL EVIDENCE FOR LARGE-SCALE SPOT ACTIVITY

ON THE PRIMARY COMPONENT OF XY URSAE MAJORIS

Edward H. Geyer

Sternwarte der Universität Bonn -

Observatorium Hoher List, D-5568 Daun, FRG

\title{
ABSTRACT
}

Extensive pe- and spectroscopic observations of the short period eclipsing binary XY UMa within the last four years confirm the star spot and stellar activity model, which was presented in 1975.

\section{INTRODUCTION AND OBSERVATIONS}

Four years agoe at the Cambridge IAU Symposium No.73 I presented a star spot model for the interpretation of the large light curve changes of the od48-day eclipsing binarY XY UMa. At that time and then in 1977 I predicted that this system should be a transient soft $X$-ray source, and a variable radio source as well (Geyer, 1976,1977a). In the last few years I carried out intensive photometric and spectroscopic observations of this EB-binary, which not only confirm the spot model but also yield a lot of new insight into the stellar activity phenomenology.

The pe-photometry in $U, B, V$ was carried out by means of a double beam photometer at the $106 \mathrm{~cm}$ - F/14.5 Cassegrain telescope of the Hoher List Observatory. This photometer allows the nearly continuous monitoring of the variable star with a typical time resolution of about 10 to 20 seconds per colour. Therefore many fine structures in the light curves become detectable, and also the time instants of the minima can be derived with an accuracy of $3 \cdot 10^{-4}$ days.

\section{THE SYSTEM PARAMETERS}

Out of the 11 light curves at my disposal since 1955, that of February / March 1976 is the most symmetric one. I used it to derive in a formal way the approximate system constants from the B- light curve: Primary minimum is a "transit"; $x=0.8$ (adopted) $; \quad k=0.54 ; r_{g}=0.41 ; r_{s}=0.22 ; i=83.5 ;$

$z=\varepsilon^{2} \sin ^{2} i=0.138 ; J_{s} / J_{g}=0.253 ; L_{s}=0.07 ; L_{g}=0.93$. 
Therefore due to its low brightness, the secondary component is invisible on blue spectrograms with dispersions of $86 \% / \mathrm{mm}$ and $136 \AA / \mathrm{mm}$. From these we derived a mass function of about 0.12 . If we adopt a mass of about one solar mass in accordance to its spectral type ( $G 2 \mathrm{~V}$ to $\mathrm{G} 5 \mathrm{~V}$ ), it follows that the mass of the secondary component corresponds to a $\mathrm{K} 5$ star. This is also conform with the above given surface brightness ratio. Though the primary component seems to be oversized by about 20\%, the system itself is still a detached one. From these facts we can conclude that the secondary component cannot be responsible for the observed light curve variations at all.

We used the calculated light curve from these system constants to obtain the residual curves from the observed light curves. These residual curves, which are freed now from the orientation and eclipsing light variations, represent the rotational variations due to asymmetric brightness distributions on the sphere of the primary component. These can now be analysed according the methods outlined already by Russell (1906) with the ambiguities that a great variety of distributions of brightness on the sphere may give rise to the same light curve. The maximum observed amplitude of the residual curve amounts to about $\mathrm{O}_{.}^{\mathrm{m}} 2$.

\section{DISCUSSION}

The observational facts for the evidence of strong spot activity on the primary component of XY UMa can be summarized as follows (figure 1):

i) Since the colour curve is in most cases strongly asymmetric during the primary minimum phase, and is oscillating around the time instant of the minimum, we have the strongest arguments for the existance of subluminous areas with reduced temperature on the stellar sphere.

ii) There exists a variable Nordmann - Tikhov effect which amounts up to 0.28 of the orbital period. It is the consequence of the described colour curve asymmetries. Thus the long debated and disbuted Nordmann - Tikhov phenomenon

(Szafraniec, 1962) finds a simple explanation by star spot activity.

iii) The observed light curve flickering and the brightness spikes, which prevail sometimes for a couple of weeks, are indicative for the existance for star spot groups rather than extended subluminous areas. Furtheron it shows that there are certainly present chromospheric and flare activities.

iv) The symmetrical light curves always occur between the appearance of asymmetric light curves. Therefore no "migrating wave" like in the typical RS CVen variables is present. v) This change over from symmetry to asymmetry repeats within 3.5 to 4 years ("spot cycle").

vi) The average system brightness of XY UMa varies on a time scale of 25 years. This is indicative for the modulation of the average spot frequency and/or the spot area on this time 

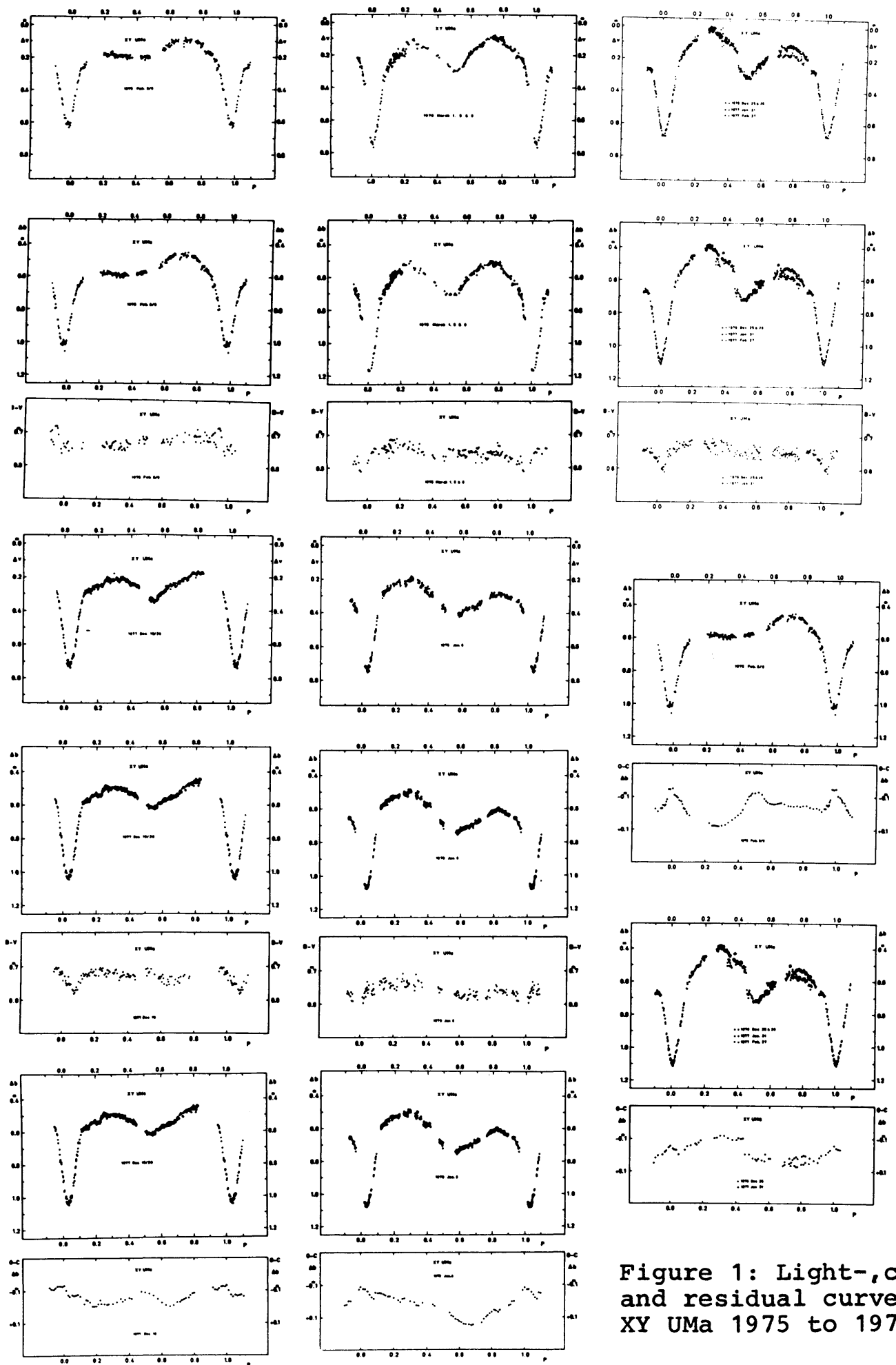

Figure 1: Light-, colourand residual curves for XY UMa 1975 to 1979 . 
scale. It may be-mere chance that the ratio of the spot cycle length to this modulation time scale is the same as in the sun. vii) The orbital period of 0.478995 day was constant during 25 years within $5 \cdot 10^{-7}$ day. In the $(0-C)$-diagram oscillations up to 28 occur (Geyer, 1977b).

viii) No circumstellar matter or matter within the system could be detected in the optical spectral range (Geyer and Metz, 1977).

\section{CONCLUDING REMARKS}

As earlier predicted, the strong stellar activity of the primary component of XY UMa should be especially prominent in the EUV spectral range and centimeter radio wavelength bands.

Finally some ideas about the evolution of such close binaries with strong magnetic star spot activities can be given: Since in analogy to the sun the spot activity is certainly connected with prominence activity (Van't Veer, 1976) loss of rotational angular momentum according to the electromagnetic coupling mechanism proposed by Schatzman (1959, 1962) for the spotted star must occur. On the other hand in a close binary system the tidal effects force its components to rotate nearly synchronous to the orbital period at least in their outer layers. Thus there exists a feedback coupling between the rotation of the components and their orbital revolution, which is not only the motor for the differential rotation of the spotted star to induce a stellar dynamo, but also for the evolution of the binary system itself. It can take place in a twofold way:

a) If the total angular momentum of the binary system is not changed by the loss of rotational angular momentum of the spotted component, then the orbital angular momentum increases with a relevant increase of the orbital period. The system becomes more detached (Geyer, 1977a).

b) On the other hand, if the total angular momentum of the binary system is decreasing by the loss of rotational angular momentum, the orbital period is shortening, and the system may finally come into contact (Van't Veer, 1979). Such a system may be the WUMa type star TZ Boo about which extensive observations have been collected by Hoffmann $(1978,1979)$.

REFERENCES

Geyer, E.H., 1976: Proc. IAU Sympos. No. 73, 313.

Geyer, E.H., 1977a: Proc. IAU Colloqu. No. 42, 292.

Geyer, E.H., 1977b: Astrophys.Space Sci. 48, 137.

Geyer, E.H. and Metz, K., 1977: Astrophys. Space Sci. 52, 351. Hoffmann, M., 1978: Astron. \& Astrophys. Suppl. 33, 63. Hoffmann, M., 1979: Private communication. Russel1, H.N., 1906: Astrophys. Jour. 24, 1. Van't Veer, F., 1976: Proc. IAU Symp. No. 73, 343. Van't Veer, F., 1979: Private communication. 


\section{DISCUSSION FOLLOWING MILONE-NAFTILAN AND GEYER}

Eaton: I have questions about your solution of the light curves of RT Lac requiring a circumstellar envelope. Doug Hall and I have published a light curve solution for the same data which does not require such an envelope. As you know, we both analyzed the same data. Your solution was based on a modified light curve in which the distortion wave was subtracted out before a solution was attempted. Hall and I carried out essentially this same procedure, obtaining your results at $\underline{\mathrm{V}}$. However the eclipse depths $(\mathrm{k} \approx 1)$ and shapes made it impossible to use this sort of model to explain the anomalous color variation of the system. Instead, we have obtained relatively consistent solutions to the $\mathrm{V}$ light curves for both 1965 and 1968-69 using a spot mode1. These require that the more massive component have an anomalous energy distribution, as indeed seems to me to be the case for your solution. How confident are you of your rectification for the photometric wave? How does your model account for the anomalous color variations?

Milone-Naftilan: The solution is only for the V light curve. The envelope would have to be gray enough to permit the observed color indices at both minima. The rectification seemed to work at both 1965 and 1968 epochs but one can never rule out that the results are somewhat compromised by the rectification procedure. A bluer star underlying the envelope is, it seems to me, a reasonable explanation. The goodness of fit of either model to the maxima is not all that great, but of course we have not yet been able to come to grips with the apparent migration of the wave, as you have been able to do.

Sadik: Have you tried to plot a magnitude-color diagram (i.e. V vs. $\overline{B-V)}$ for out-of-eclipses points, that is to see if the system is getting redder or bluer when it's brighter? And secondly, is the V vs. $B-V$ variation in the same sense from cycle to cycle or from one light curve to another?

Paku11: How do you solve your highly asymmetric light curves?

Geyer: The system parameters were obtained in the usual formal way from the most symmetric light curve I had observed. These parameters served to calculate the "theoretical light curve", which was used to obtain the residual light curves from the observed ones. In this way the light variation by orientation and eclipse is more or less removed, and the residual curve gives the intrinsic light variations due to stellar spots.

van ' $t$ Veer: I agree with you for the importance of prominence activity involving angular momentum loss and change of orbital parameters. I do not believe the detectability of the Tikhman-Nordhoff effect in your observations. The dispersion of the interstellar medium is insufficient to explain phase shifts of the light curve for different colors. 